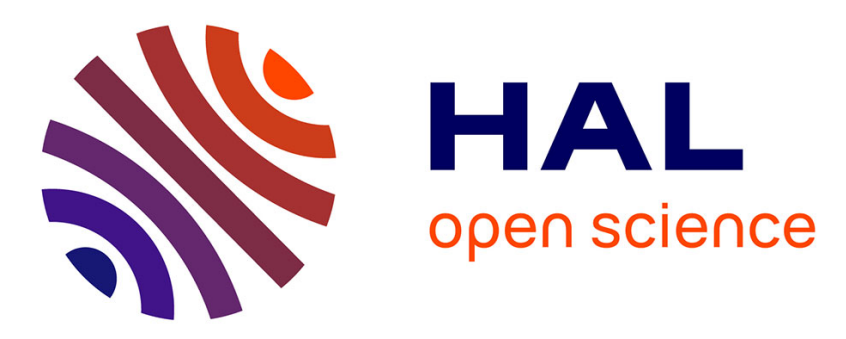

\title{
Sequent Calculi for Nominal Tense Logics: A Step Towards Mechanization?
}

Stéphane Demri

\section{To cite this version:}

Stéphane Demri. Sequent Calculi for Nominal Tense Logics: A Step Towards Mechanization?. International Conference on Automated Reasoning with Analytic Tableaux and Related Methods (TABLEAUX'99), Jun 1999, Saratoga Springs, United States. pp.140-155, 10.1007/3-540-487549_15. hal-03195331

\section{HAL Id: hal-03195331 https://hal.science/hal-03195331}

Submitted on 11 Apr 2021

HAL is a multi-disciplinary open access archive for the deposit and dissemination of scientific research documents, whether they are published or not. The documents may come from teaching and research institutions in France or abroad, or from public or private research centers.
L'archive ouverte pluridisciplinaire $\mathbf{H A L}$, est destinée au dépôt et à la diffusion de documents scientifiques de niveau recherche, publiés ou non, émanant des établissements d'enseignement et de recherche français ou étrangers, des laboratoires publics ou privés. 


\title{
Sequent Calculi for Nominal Tense Logics: A Step Towards Mechanization?
}

\author{
Stéphane Demri \\ Laboratoire LEIBNIZ- U.M.R. 5522 \\ 46 Avenue Felix Viallet, 38031 Grenoble, France \\ Email: demri@imag.fr
}

\begin{abstract}
We define sequent-style calculi for nominal tense logics characterized by classes of modal frames that are first-order definable by certain $\Pi_{1}^{0}$-formulae and $\Pi_{2}^{0}$-formulae. The calculi are based on d'Agostino and Mondadori's calculus KE and therefore they admit a restricted cutrule that is not eliminable. A nice computational property of the restriction is, for instance, that at any stage of the proof, only a finite number of potential cut-formulae needs to be taken under consideration. Although restrictions on the proof search (preserving completeness) are given in the paper and most of them are theoretically appealing, the use of those calculi for mechanization is however doubtful. Indeed, we present sequent calculi for fragments of classical logic that are syntactic variants of the sequent calculi for the nominal tense logics.
\end{abstract}

\section{Introduction}

Background. The nominal tense logics are extensions of Prior tense logics (see e.g. [Pri57,RU71]) by adding nominals (also called names) to the language (see e.g. [Bla93]). Nominals are understood as atomic propositions that hold true in a unique world of the Kripke-style models. The nominal tense logics are quite expressive since not only do they extend the standard (mono)modal logics by adding a past operator (giving the tense flavour) but they also admit nominals in the language. In spite of the analogy between nominals (in the object language of the logic) and prefixes, also called labels, used in various proof systems for modal logics (see e.g. [Fit83,Wal90]), no proof systems for nominal tense logics using this conceptual similarity exist. This is all the more surprising because during the last years, prefixed calculi have regained some interest (see e.g. [Ogn94,Mas94,Gov95,Rus96,Gab96,BMV97,BG97]).

Although designing general frameworks defining proof systems for modal logics is a fundamental task, other works deal with the difficult problem of improving significantly the mechanization of logics by finding refined properties, mostly proof-theoretical, that provide better complexity bounds or that allow the design of efficient decision procedures (see e.g. [Hud96,GHM98,Heu98]). We claim that the latter approach is the most promising for mechanization. As witness, the present paper illustrates that for many nominal tense logics, it is not so difficult to find a general framework for mechanization as long as only qualitative properties (soundness, completeness, ...) are investigated.

Our contribution. For any nominal tense logic $\mathcal{L}$ from the class $\mathcal{C}_{\Pi_{2}^{0}}$ defined 
in this paper, we define a sequent-style calculus, say $G \mathcal{L}$, that is based on the sequent-style counterpart of the calculus KE defined in [dM94]. Our calculi admit a cut rule satisfying the following nice computational properties. When reading the proof upwards, at any stage of the construction of the proof,

(CR1) the number of potential cut-formulae is linear in the size of the part of the proof constructed so far;

(CR2) any potential cut-formula can be computed in linear-time in the size of the part of the proof constructed so far.

(CR3) the size of any potential cut-formula is linear in the size of the part

of the proof constructed so far (consequence of (CR2));

(CR1) means for instance that when growing the proofs upwards, if one decides to apply the cut rule at some stage, only a limited amount of candidate cut formulae could be useful to end the construction of the proof. The non-determinism of the cut rule is therefore weakened. Analycity is however not guaranteed because new nominals shall be introduced during the construction of the proofs. It is known (see e.g. [Boo84,dM94]) that cut-elimination is not always a guarantee for (efficient) mechanization. The search for some analytic cut rule is often desirable and the calculi defined in this paper follow that line of research. Furthermore, we take advantage of the presence of nominals in the modal language to use "implicit prefixes" in the proof systems. As far as we know, the idea of using such implicit prefixes when nominals are involved is due to Konikowska [Kon97]. In [Kon97], Rasiowa-Sikorski-style calculi for relative similarity logics are defined. Herein, we generalize the use of implicit prefixes to a class of nominal tense logics and we introduce various restrictions on the applications of the rules while preserving completeness. Although, for some particular logics, decision procedures can be obtained using the restrictions, in the general case, the design of decision procedures (when possible) is not straightforward from our calculi. It is also fair to state that the paper [Kon97] has been a source of inspiration in order to develop some of the ideas present in this paper.

In the last part of the paper, we define sequent-style calculi (based on KEQ [d'A90]), say $G F O L_{\mathcal{L}}$, for fragments of the classical logic such that the calculi $G \mathcal{L}$ and $G F O L_{\mathcal{L}}$ can be clearly viewed as syntactic variants. This allows to observe that $G \mathcal{L}$ is first-order in nature and to explain why it is so. Moreover, it clearly raises the questions about the relevancy of defining calculi within a general prooftheoretical framework when mechanization is wanted. Apart from the technical results of the paper, we wish to formally illustrate why numerous calculi for modal logics can be viewed as an encoding into classical logic. Although this fact is widely recognized for particular systems, we want here to propose a more general picture since the class $\mathcal{C}_{\Pi_{2}^{0}}$ is quite large.

Related work. Most of the proof systems designed for nominals tense logics are Hilbert-style ones [Bla93]. Calculi for (non nominal) tense logics can be for instance found in [RU71,Kra96,Heu98,BG98] but these calculi do not treat the nominal case and they do not consider so large a class of logics as $\mathcal{C}_{\Pi_{2}^{0}}$. In [DG99], display calculi for nominal tense logics have been defined and cut is not only eliminable but also a strong normalization theorem is established. For all the calculi designed in the present paper, cut (or equivalently the principle of bivalence) is not eliminable. Furthermore, the sequent calculi defined in the present paper are based on a completely different approach: we rather use the nominals as "implicit prefixes". In that sense, our calculi are explicit systems 
following [Gor99] but without introducing any extra proof-theoretical device that does not belong to the object modal language. Furthermore, the calculi defined in this paper does not differ very much in spirit with those defined in [Rus96,BMV98]. Indeed, we associate syntactically rules to formulas defining relational theories. However, we are able to capture all the conditions on frames for the properly displayable modal logics defined in [Kra96]. We wish also to thank one of the referees for pointing us to [Bla98,Tza99] where tableau-style calculi having technical similarities with ours have been defined.

\section{Nominal tense logics}

Given a countably infinite set $^{1}$ For $_{0}=\left\{\mathrm{p}_{0}, \mathrm{p}_{1}, \mathrm{p}_{2}, \ldots\right\}$ of atomic propositions and a countably infinite set For $_{0}^{N}=\left\{\dot{i}_{0}, \dot{i}_{1}, \ldots\right\}$ of names, the formulas $\phi \in$ $\operatorname{NTL}(G, H)$ are inductively defined as follows: $\phi::=\mathrm{p}_{k}\left|i_{k}\right| \phi_{1} \wedge \phi_{2} \mid$ $\phi_{1} \Rightarrow \phi_{2}|\neg \phi| G \phi \mid H \phi$ for $\mathrm{p}_{k} \in$ For $_{0}$ and $i_{k} \in$ For $_{0}^{N}$. Standard abbreviations include $\Leftrightarrow, F, P$. We write $|\phi|$ to denote the length of the formula $\phi$ for some (unspecified) succinct encoding. An occurrence of the formula $\psi$ is said to be a subformula of the formula $\phi$ of secondary disjunctive force $\stackrel{\text { def }}{\Leftrightarrow} \psi$ is a subformula of $\phi$ and $\psi$ is the immediate subformula either of a conjunction in $\phi$ of negative polarity or of an implication in $\phi$ of positive polarity. We use here the usual notion of polarity. For instance, $\mathrm{p}_{0}$ occurs negatively in $\left(\mathrm{p}_{1} \wedge \mathrm{p}_{0}\right) \Rightarrow \mathrm{p}_{1}$. A modal frame $\mathcal{F}=\langle W, R\rangle$ is a pair such that $W$ is a non-empty set and $R$ is a binary relation on $W$. We use $R(w) \stackrel{\text { def }}{=}\{v \in W:(w, v) \in R\}$. A model $\mathcal{M}$ is a structure $\mathcal{M}=\langle W, R, m\rangle$ such that $\langle W, R\rangle$ is a frame and $m$ is a mapping $m:$ For $_{0} \cup$ For $_{0}^{N} \rightarrow \mathcal{P}(W)$ where for any $i \in$ For $_{0}^{N}, m(i)$ is a singleton. Let $\mathcal{M}=$ $\langle W, R, m\rangle$ be a model and $w \in W$. The formula $\phi$ is satisfied by the world $w \in W$ in $\mathcal{M} \stackrel{\text { def }}{\Leftrightarrow} \mathcal{M}, w \models \phi$ where the satisfaction relation $\models$ is inductively defined as follows: $\mathcal{M}, w \models \mathrm{p} \stackrel{\text { def }}{\Leftrightarrow} w \in m(\mathrm{p})$, for every $\mathrm{p} \in \operatorname{For}_{0} \cup \operatorname{For}_{0}^{N} ; \mathcal{M}, w \models G \phi \stackrel{\text { def }}{\Leftrightarrow}$ for every $w^{\prime} \in R(w), \mathcal{M}, w^{\prime} \models \phi ; \mathcal{M}, w \models H \phi \stackrel{\text { def }}{\Leftrightarrow}$ for every $w^{\prime} \in R^{-1}(w)$, $\mathcal{M}, w^{\prime} \models \phi\left(R^{-1}\right.$ is the converse of $\left.R\right)$. We omit the standard conditions for the propositional connectives. A formula $\phi$ is true in a model $\mathcal{M}$ (written $\mathcal{M} \models \phi$ ) $\stackrel{\text { def }}{\Leftrightarrow}$ for every $w \in W, \mathcal{M}, w \models \phi$. A formula $\phi$ is true in a frame $\mathcal{F}$ (written $\mathcal{F}=\phi) \stackrel{\text { def }}{\Leftrightarrow} \phi$ is true in every model based on $\mathcal{F}$. In what follows, by a logic $\mathcal{L}$ we understand a pair $\langle\operatorname{NTL}(H, G), \mathcal{C}\rangle$ where $\mathcal{C}$ is a non-empty class of modal frames. A formula $\phi$ is said to be $\mathcal{L}$-valid $\stackrel{\text { def }}{\Leftrightarrow} \phi$ is true in all the models based on the frames of $\mathcal{C}$. A formula $\phi$ is said to be $\mathcal{L}$-satisfiable $\stackrel{\text { def }}{\Leftrightarrow} \neg \phi$ is not $\mathcal{L}$-valid. Now, we define the class $\mathcal{C}_{\Pi_{2}^{0}}$ of nominal tense logics announced in the introduction. First, we need to present preliminary definitions. Here, we consider the fragment of FOL built using the following vocabulary: $T$ is the verum logical constant; $\left\{\mathrm{P}_{k}: k \in \omega\right\}$ is a countable set of unary predicate symbols; $\mathrm{R}$ and = (identity) are the unique

\footnotetext{
${ }^{1}$ The metavariables for atomic propositions [resp. for nominals] are $\mathrm{p}, \mathrm{q}, \ldots$ [resp. $i, j, \ldots]$. When $p$ [resp. $i]$ is subscripted by some natural number, we mean exactly the members from For 0 [resp. from For $\left._{0}^{N}\right]$.
} 
binary predicate symbols; $\left\{\mathrm{a}_{k}: k \in \omega\right\}$ is a countable set $^{2}$ of individual constants; $\left\{\mathrm{x}_{k}: k \in \omega\right\} \cup\left\{\mathrm{y}_{k}: k \in \omega\right\}$ is a countable set of individual variables. A $\Pi_{1}^{0}$ formula is a FOL-formula of the form $\forall \mathrm{x}_{1} \ldots \forall \mathrm{x}_{n} \phi$ where $\phi$ is quantifier-free and $n \geq 1$. A $\Pi_{2}^{0}$-formula is a FOL-formula of the form $\forall \mathrm{x}_{1} \ldots \forall \mathrm{x}_{n} \quad \exists \mathrm{y}_{1} \ldots \exists \mathrm{y}_{m} \phi$ where $\phi$ is quantifier-free and $n, m \geq 1$. A restricted $\Pi_{2}^{0}$-formula $\psi$ is defined here as a FOL-formula of the form $\forall \mathrm{x}_{1} \ldots \forall \mathrm{x}_{n} \exists \mathrm{y}_{1} \ldots \exists \mathrm{y}_{m}\left(\phi_{1} \Rightarrow \phi_{2}\right)$ where

1. $\psi$ is in prenex normal form (PNF) and $\phi_{1} \Rightarrow \phi_{2}$ is precisely its matrix;

2. $\phi_{1}$ and $\phi_{2}$ are formulas built upon the binary predicate symbols $\mathrm{R},=$, the truth logical constant $T$ and from $\left\{\mathrm{x}_{1}, \ldots, \mathrm{x}_{n}, \mathrm{y}_{1}, \ldots, \mathrm{y}_{m}\right\}$ (no individual constant occurs in $\left.\phi_{1} \Rightarrow \phi_{2}\right) ; n \geq 1 ; m \geq 0$;

3. $\phi_{1}$ is either the logical constant $\bar{T}$ or a finite conjunction of literals (atomic formulae or negated atomic formulae) where no $\mathrm{y}_{i}$ occurs in $\phi_{1}$;

4. $\phi_{2}$ is a disjunction of conjunctions of literals.

A nominal tense $\operatorname{logic} \mathcal{L}=\langle\operatorname{NTL}(G, H), \mathcal{C}\rangle$ is an element of the class $\mathcal{C}_{\Pi_{2}^{0}} \stackrel{\text { def }}{\Leftrightarrow}$ there is a $\operatorname{set}^{3} \Phi$ of restricted $\Pi_{2}^{0}$-formulae such that $\mathcal{C}$ is exactly the set of frames satisfying each formula from $\Phi$ (in the first-order sense). The class $\mathcal{C}$ of modal frames is also said to be $\mathcal{C}_{\Pi_{2}^{0}}$-definable. The class $\mathcal{C}_{\Pi_{2}^{0}}$ is quite large. By manipulation at the first-order level one can show:

1. For any closed (unrestricted) $\Pi_{2}^{0}$-formula $\psi \stackrel{\text { def }}{=} \forall \mathrm{x}_{1} \ldots \forall \mathrm{x}_{n} \exists \mathrm{y}_{1} \ldots \exists \mathrm{y}_{m}\left(\phi_{1} \Rightarrow\right.$ $\left.\phi_{2}\right)$ in PNF such that the only variables in $\phi_{1}$ belong to $\left\{\mathrm{x}_{1}, \ldots, \mathrm{x}_{n}\right\}$, there exists a finite conjunction of restricted $\Pi_{2}^{0}$-formulae equivalent to $\psi$.

2. Every primitive first-order formula in the sense of [Kra96] is logically equivalent to a restricted $\Pi_{2}^{0}$-formula.

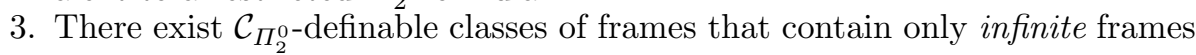
(see e.g. [Bla93]).

Expressivity of the restricted $\Pi_{2}^{0}$-formulae is also well-illustrated by the fact that not only are there $\mathcal{C}_{\Pi_{2}^{0}}$-definable classes of frames that are not modally definable but also all the first-order classes of frames defined by a conjunction of conditions from Figure 2 and Figure 3 in [Gor99] are $\mathcal{C}_{\Pi_{2}^{0}}$-definable. All the first-order definable classes of frames considered in [Rus96,CFdCGH97] are $\mathcal{C}_{\Pi_{2}^{0-}}$ definable and $\mathcal{C}_{\Pi_{2}^{0}}$ contains all the modal logics (in their nominal tense version) defined with Horn clauses from [BMV98]. Furthermore, for any nominal tense $\operatorname{logic} \mathcal{L}=\langle\operatorname{NTL}(G, H), \mathcal{C}\rangle$ such that $\mathcal{C}$ is first-order definable by a finite set $\Phi$ of restricted $\Pi_{2}^{0}$-formulae, it is known that the $\mathcal{L}$-validity problem can be translated into FOL-validity (using [Ben83,GG93]). However, there is no guarantee that $\mathcal{L}$ admits a proof system (based on KE for instance) such that the cut rule satisfies the conditions (CR1), (CR2) and (CR3) -see Section 1. In the present paper, the delimitations of the class $\mathcal{C}_{\Pi_{2}^{0}}$ has been designed in such a way that the sequent calculi (based on KE) admit a cut-rule satisfying the computationnally nice conditions (CR1), (CR2) and (CR3) -other restrictions on the applications of various rules shall be introduced. Those criteria distinguish our work from

\footnotetext{
${ }^{2}$ The metavariables for individual constants [resp. for individual variables] are a, b, . . $[$ resp. $\mathrm{x}, \mathrm{y}, \ldots]$. When a [resp. $\mathrm{x}$ and $\mathrm{y}]$ are subscripted by some natural numbers we mean exactly the members from $\left\{\mathrm{a}_{k}: k \in \omega\right\}$ [resp. from $\left.\left\{\mathrm{x}_{k}: k \in \omega\right\} \cup\left\{\mathrm{y}_{k}: k \in \omega\right\}\right]$.

${ }^{3} \Phi$ should be understood as a (possibly infinite) conjunction.
} 
the standard translation into FOL but other criteria are of course possible as done in [BMV97, Section 4] where enlighting analyses about the behaviour of the falsum $\perp$ can be found. To conclude this section, we warn the reader that although $\mathcal{C}_{\Pi_{2}^{0}}$ is undoubtly a very large class, we ignore whether it contains any logic useful in practice.

\section{Sequent-style calculi for nominal tense logics}

In this section, $\mathcal{L}$ denotes a nominal tense logic $\langle\operatorname{NTL}(G, H), \mathcal{C}\rangle$ in $\mathcal{C}_{\Pi_{2}^{0}}$ characterized by the set $\Phi$ of restricted $\Pi_{2}^{0}$-formulae.

\subsection{Preliminaries}

Most of the prefixed tableaux calculi for modal logics use prefixes as a compact way to represent sets of positive literals in first-order logic. It partly explains why numerous calculi can be viewed as a "clever translation" 4 into classical logic (see e.g. [Gen92]). For instance, in [Fit83], a prefix is defined as a (non-empty) sequence of natural numbers. A sequence $i_{1} \ldots i_{n} \in \omega^{*}(n \geq 1)$ can be understood (for example for the modal logic S4) as the $\operatorname{set}^{5}\left\{\mathrm{R}\left(\mathrm{a}_{i_{1} \ldots i_{m}}, \mathrm{a}_{i_{1} \ldots i_{m^{\prime}}}\right): 1 \leq m \leq\right.$ $\left.m^{\prime} \leq n\right\}$ of positive literals (the $\mathrm{a}_{\sigma}$ 's are individual constants). It is therefore inaccurate to believe that since prefixes can be interpreted at the metalevel by worlds, then prefixes and nominals have the same expressive power. Actually, the prefixes are more expressive since the nominals do not contain any information about the accessibility relation. However, formulas involving nominals can encode first-order literals, positive and negative ones as shown below. For any model $\mathcal{M}=\langle W, R, m\rangle$, it is easy to show that $i_{1} \Rightarrow G \neg i_{2}$ is true in $\mathcal{M}$ iff $\left(m\left(i_{1}\right), m\left(i_{2}\right)\right) \notin R$. So, $i_{1} \Rightarrow G \neg i_{2}$ can be used as a negative literal. What seems to be lost here, is a conciseness of the representation: each literal is represented by one nominal tense formula of the same length (modulo some constant) and it is the approach chosen in the calculi defined in Section 3.2. However, since we are dealing with logics whose satisfiability is NP-hard, the following argument shows that conciseness is a secondary issue for mechanization. Indeed, let $i_{1} \ldots i_{n}$ be a (non-empty) sequence of natural numbers representing a set $X$ of first-order positive literals subset of $\left\{\mathrm{R}\left(\mathrm{a}_{i_{1} \ldots i_{m}}, \mathrm{a}_{i_{1} \ldots i_{m^{\prime}}}\right): m, m^{\prime} \in\{1, \ldots, n\}\right\}$ (it depends on the modal logic we consider but let us treat the general case). The length of $i_{1} \ldots i_{n}$, say $\left|i_{1} \ldots i_{n}\right|$, is naturally defined as the sum of the length (in binary writing) of each natural number occurring in $i_{1} \ldots i_{n}$. For instance, $\left|i_{1} \ldots i_{n}\right| \geq n$ and $\operatorname{card}(X) \leq n^{2}$. Let $\phi_{X}$ be the following nominal tense formula $\bigwedge_{\mathrm{R}\left(\mathrm{a}_{i_{1} \ldots i_{m}}, \mathrm{a}_{i_{1} \ldots i_{m^{\prime}}}\right) \in X} \dot{i}_{i_{1} \ldots i_{m}} \Rightarrow \neg G \neg \dot{i}_{i_{1} \ldots i_{m^{\prime}}}$ that encodes the prefix $i_{1} \ldots i_{n}$ (or equivalently that encodes $X)$. The generalized conjunction $\bigwedge$ should be here understood as an abbreviation for a certain amount of binary conjunctions. $\left|\phi_{X}\right|$ is in $\mathcal{O}\left(\left|i_{1} \ldots i_{n}\right|^{3}\right)$ and therefore, if a formula $\phi$ has a proof $\Pi$ with the "concise representation" of the positive literals, then $\phi$ has a proof $\Pi^{\prime}$ with the representation of literals "in extension" where $\left|\Pi^{\prime}\right|$ is in $\mathcal{O}\left(|\Pi|^{3}\right)$. The length of the

\footnotetext{
${ }^{4}$ [BG97] is one of the rare papers where such a relationship is explicitly recognized.

${ }^{5}$ Since $\omega^{*}$ and $\omega$ have the same cardinality, without any loss of generality, we can assume that the individual constants and the nominals are respectively of the form $\mathrm{a}_{\sigma}$ and $\mathbf{i}_{\sigma}$ where $\sigma \in \omega^{*}$.
} 
proof $\Pi$, denoted $|\Pi|$, is defined as the number of nodes in the tree. In a more general setting, it would be necessary to use a more refined definition of proof complexity which takes into account the length of proof steps. Since the calculi involved in the paper use a very restricted cut-rule (the size of the cut-formula is linear in the size of the conclusion), our definition is sufficient for our needs. As no subexponential algorithm for any NP-hard problem is known, such a cubic overhead $\left(\left|\phi_{X}\right| \in \mathcal{O}\left(\left|i_{1} \ldots i_{n}\right|^{3}\right)\right)$ is not so significant (even in the worst-case) when dealing with NP-hard problems (and a fortiori with PSPACE-hard problems). Of course, this is highly significant to establish tight complexity upper bounds as done in [Hud96]. In [Kri63,CFdCGH97] and [Heu98, Chapter 4], some of the graphical representations of the sets of (positive) first-order literals enjoy some conciseness property comparable to the one for prefixes.

\subsection{Definition}

The basic syntactic objects in the calculus are sequents. A sequent is an expression of the form $\Gamma \vdash \Delta$ where $\Gamma$ and $\Delta$ are finite multisets of nominal tense formulae, i.e. unordered collections of formulae that may contain several occurrences of the same formula. We write $\phi$ for $\{\phi\}$ and let "," denote the multiset union. The length of the sequent $\Gamma \vdash \Delta$, denoted $|\Gamma \vdash \Delta|$, is the sum of the length of each element from $\Gamma, \Delta$. The sequent calculus, say $G \mathcal{L}$, for the logic $\mathcal{L}$ contains the rules in Figures 1-3. Other rules depending on $\Phi$ are presented when needed. In Figure 2, the rules (refl), (sym) and (trans) encode properties of identity (reflexivity, symmetry and transitivity). Similarly, the rules $(s u b \vdash)$ and $(\vdash s u b)$ ("sub" stands for substitution) encode that identical terms can be substituted. The (start)-rule has a special status since in any proof, this rule is applied exactly once, at the root (with the forthcoming restriction $\left(R_{\text {start }}\right)$ ). This initiates the introduction of nominals that behave as prefixes. Observe that $i \Rightarrow \phi$ is $\mathcal{L}$-valid iff $\phi$ is $\mathcal{L}$-valid when $i$ does not occur in $\phi$.

$$
\Gamma, \mathbf{i} \Rightarrow \psi \vdash \Delta, \mathbf{i} \Rightarrow \psi\left(\text { initial sequents) } \frac{\vdash \mathrm{i} \Rightarrow \phi}{\vdash \phi}(\text { start })\right.
$$

For the (start)-rule, i does not occur in $\phi$.

Fig. 1. Initial sequents and the rule (start)

We continue here the definition of $G \mathcal{L}$. Let $\sigma$ be a finite sequence of formulas of the form $\mathrm{i} \Rightarrow \mathrm{j}$, $\mathrm{i} \Rightarrow \neg G \neg j, \mathrm{i} \Rightarrow \neg \mathrm{j}, \mathrm{i} \Rightarrow G \neg \mathrm{j}$. Those formulae precisely "encodes" positive and negative first-order literals whose (binary) predicate symbol is either $=$ or $\mathrm{R}$. We define the sequent $(\Gamma \vdash \Delta) \otimes \sigma$ inductively on the length of $\sigma$ as follows ( $\lambda$ denotes the empty string and $\otimes$ is simply an operator that inserts formulae in sequents):

$$
\begin{aligned}
& -(\Gamma \vdash \Delta) \otimes \lambda \stackrel{\text { def }}{=} \Gamma \vdash \Delta ; \\
& -(\Gamma \vdash \Delta) \otimes(i \Rightarrow j) \cdot \sigma^{\prime} \stackrel{\text { def }}{=}(\Gamma, i \Rightarrow j \vdash \Delta) \otimes \sigma^{\prime} ; \\
& -(\Gamma \vdash \Delta) \otimes(i \Rightarrow G \neg j) \cdot \sigma^{\prime} \stackrel{\text { def }}{=}(\Gamma, i \Rightarrow G \neg j \vdash \Delta) \otimes \sigma^{\prime} ;
\end{aligned}
$$




$$
\begin{gathered}
\frac{\Gamma, \mathrm{i} \Rightarrow \phi \vdash \Delta}{\Gamma \vdash \Delta, \mathrm{i} \Rightarrow \neg \phi}(\vdash \neg) \quad \frac{\Gamma \vdash \Delta, \mathrm{i} \Rightarrow \phi}{\Gamma, \mathrm{i} \Rightarrow \neg \phi \vdash \Delta}(\neg \vdash) \quad \frac{\Gamma, \mathrm{i} \Rightarrow \phi_{1} \vdash \Delta, \mathrm{i} \Rightarrow \phi_{2}}{\Gamma \vdash \Delta, \mathrm{i} \Rightarrow\left(\phi_{1} \Rightarrow \phi_{2}\right)}(\vdash \Rightarrow) \\
\frac{\Gamma, \mathrm{i} \Rightarrow \phi_{1}, \mathrm{i} \Rightarrow \phi_{2} \vdash \Delta}{\Gamma, \mathrm{i} \Rightarrow \phi_{1}, \mathrm{i} \Rightarrow\left(\phi_{1} \Rightarrow \phi_{2}\right) \vdash \Delta}(\Rightarrow \vdash)_{1} \quad \frac{\Gamma \vdash \Delta, \mathrm{i} \Rightarrow \phi_{1}, \mathrm{i} \Rightarrow \phi_{2}}{\Gamma, \mathrm{i} \Rightarrow\left(\phi_{1} \Rightarrow \phi_{2}\right) \vdash \Delta, \mathrm{i} \Rightarrow \phi_{2}}(\Rightarrow \vdash)_{2} \\
\frac{\Gamma, \mathrm{i} \Rightarrow \phi \vdash \Delta, \mathrm{i} \Rightarrow \psi}{\Gamma, \mathrm{i} \Rightarrow \phi \vdash \Delta, \mathrm{i} \Rightarrow(\phi \wedge \psi)}(\vdash \wedge)_{1} \quad \frac{\Gamma, \mathrm{i} \Rightarrow \psi \vdash \Delta, \mathrm{i} \Rightarrow \phi}{\Gamma, \mathrm{i} \Rightarrow \psi \vdash \Delta, \mathrm{i} \Rightarrow(\phi \wedge \psi)}(\vdash \wedge)_{2} \\
\frac{\Gamma, \mathrm{i} \Rightarrow \phi, \mathrm{i} \Rightarrow \psi \vdash \Delta}{\Gamma, \mathrm{i} \Rightarrow(\phi \wedge \psi) \vdash \Delta}(\wedge \vdash) \\
\frac{\Gamma, \mathrm{i} \Rightarrow G \psi, \mathrm{j} \Rightarrow \psi \vdash \Delta, \mathrm{i} \Rightarrow G \neg \mathrm{j}}{\Gamma, \mathrm{i} \Rightarrow G \psi \vdash \Delta, \mathrm{i} \Rightarrow G \neg \mathrm{j}}(G \vdash) \quad \frac{\Gamma \vdash \Delta, \mathrm{j} \Rightarrow \psi, \mathrm{i} \Rightarrow G \neg \mathrm{j}}{\Gamma \vdash \Delta, \mathrm{i} \Rightarrow G \psi}(\vdash G) \\
\frac{\Gamma, \mathrm{j} \Rightarrow H \psi, \mathrm{i} \Rightarrow \psi \vdash \Delta, \mathrm{i} \Rightarrow G \neg \mathrm{j},}{\Gamma, \mathrm{j} \Rightarrow H \psi \vdash \Delta, \mathrm{i} \Rightarrow G \neg \mathrm{j}}(H \vdash) \quad \frac{\Gamma \vdash \Delta, \mathrm{j} \Rightarrow \psi, \mathrm{j} \Rightarrow G \neg \mathrm{i}}{\Gamma \vdash \Delta, \mathrm{i} \Rightarrow H \psi}(\vdash H)
\end{gathered}
$$

in $(\vdash H)$ and $(\vdash G)$, j does not occur in the conclusion

$$
\begin{gathered}
\frac{\Gamma, \mathrm{i} \Rightarrow \mathrm{j}, \mathrm{i}^{\prime} \Rightarrow \mathrm{j}, \mathrm{i} \Rightarrow \mathrm{i}^{\prime} \vdash \Delta}{\Gamma, \mathrm{i} \Rightarrow \mathrm{j}, \mathrm{i}^{\prime} \Rightarrow \mathrm{j} \vdash \Delta}(N O M=\vdash) \\
\frac{\Gamma, \mathrm{i} \Rightarrow \psi \vdash \Delta, \mathrm{i}^{\prime} \Rightarrow \psi, \mathrm{i} \Rightarrow \mathrm{i}^{\prime}}{\Gamma, \mathrm{i} \Rightarrow \psi \vdash \Delta, \mathrm{i}^{\prime} \Rightarrow \psi}(\vdash N O M=) \\
\frac{\Gamma, \mathrm{i} \Rightarrow G \psi, \mathrm{i} \Rightarrow G \neg \mathrm{i}^{\prime} \vdash \Delta, \mathrm{i}^{\prime} \Rightarrow \psi}{\Gamma, \mathrm{i} \Rightarrow G \psi \vdash \Delta, \mathrm{i}^{\prime} \Rightarrow \psi}\left(N O M_{G} \vdash\right) \\
\frac{\Gamma, \mathrm{i} \Rightarrow \mathrm{j} \vdash \Delta, \mathrm{i}^{\prime} \Rightarrow G \neg \mathrm{j}, \mathrm{i}^{\prime} \Rightarrow G \neg \mathrm{i}}{\Gamma, \mathrm{i} \Rightarrow \mathrm{j} \vdash \Delta, \mathrm{i}^{\prime} \Rightarrow G \neg \mathrm{j}}\left(\vdash N O M_{G}\right) \\
\frac{\Gamma, \mathrm{i} \Rightarrow H \psi, \mathrm{i}^{\prime} \Rightarrow G \neg \mathrm{i} \vdash \Delta, \mathrm{i}^{\prime} \Rightarrow \psi}{\Gamma, \mathrm{i} \Rightarrow H \psi \vdash \Delta, \mathrm{i}^{\prime} \Rightarrow \psi}\left(N O M_{H} \vdash\right) \\
\frac{\Gamma, \mathrm{i} \Rightarrow \mathrm{j} \vdash \Delta, \mathrm{i}^{\prime} \Rightarrow H \neg \mathrm{j}, \mathrm{i} \Rightarrow G \neg \mathrm{i}^{\prime}}{\Gamma, \mathrm{i} \Rightarrow \mathrm{j} \vdash \Delta, \mathrm{i}^{\prime} \Rightarrow H \neg \mathrm{j}}\left(\vdash N O M_{H}\right) \\
\frac{\Gamma, \mathrm{j} \Rightarrow \mathrm{i}, \mathrm{i} \Rightarrow \mathrm{j} \vdash \Delta}{\Gamma, \mathrm{i} \Rightarrow \mathrm{j} \vdash \Delta}(s y m) \frac{\Gamma, \mathrm{i} \Rightarrow \mathrm{k}, \mathrm{i} \Rightarrow \mathrm{j}, \mathrm{j} \Rightarrow \mathrm{k} \vdash \Delta}{\Gamma, \mathrm{i} \Rightarrow \mathrm{j}, \mathrm{j} \Rightarrow \mathrm{k} \vdash \Delta}(\text { trans }) \\
\frac{\Gamma, \mathrm{i} \Rightarrow \phi, \mathrm{i} \Rightarrow \mathrm{j}, \mathrm{j} \Rightarrow \phi \vdash \Delta}{\Gamma, \mathrm{i} \Rightarrow \mathrm{j}, \mathrm{j} \Rightarrow \phi \vdash \Delta}(s u b \vdash) \quad \frac{\Gamma, \mathrm{i} \Rightarrow \mathrm{j} \vdash \Delta, \mathrm{i} \Rightarrow \phi, \mathrm{j} \Rightarrow \phi}{\Gamma, \mathrm{i} \Rightarrow \mathrm{j} \vdash \Delta, \mathrm{j} \Rightarrow \phi}(\vdash s u b) \\
\frac{\Gamma, \mathrm{j} \Rightarrow \mathrm{j}^{\prime}, \mathrm{i} \Rightarrow G \neg \mathrm{j}, \mathrm{i} \Rightarrow G \neg \mathrm{j}^{\prime} \vdash \Delta}{\Gamma, \mathrm{j} \Rightarrow \mathrm{j}^{\prime}, \mathrm{i} \Rightarrow G \neg \mathrm{j} \vdash \Delta}\left(s u b^{\prime} \vdash\right) \\
\frac{\Gamma, \mathrm{j} \Rightarrow \mathrm{j}^{\prime} \vdash \Delta, \mathrm{i} \Rightarrow G \neg \mathrm{j}, \mathrm{i} \Rightarrow G \neg \mathrm{j}^{\prime}}{\Gamma, \mathrm{j} \Rightarrow \mathrm{j}^{\prime} \vdash \Delta, \mathrm{i} \Rightarrow G \neg \mathrm{j}}\left(\vdash s u b^{\prime}\right)
\end{gathered}
$$

Fig. 2. Common core of (introduction) rules in $G \mathcal{L}$ 
$\frac{\Gamma \vdash \Delta, \mathrm{i} \Rightarrow \psi \quad \Gamma, \mathrm{i} \Rightarrow \psi \vdash \Delta}{\Gamma \vdash \Delta}(P B)$

Fig. 3. Principle of bivalence

$-(\Gamma \vdash \Delta) \otimes(i \Rightarrow \neg j) \cdot \sigma^{\prime} \stackrel{\text { def }}{=}(\Gamma \vdash \Delta, i \Rightarrow j) \otimes \sigma^{\prime} ;$

$-(\Gamma \vdash \Delta) \otimes(i \Rightarrow \neg G \neg j) \cdot \sigma^{\prime} \stackrel{\text { def }}{=}(\Gamma \vdash \Delta, i \Rightarrow G \neg j) \otimes \sigma^{\prime}$.

Let $\psi$ be a restricted $\Pi_{2}^{0}$-formula of the form

$$
\begin{gathered}
\forall \mathrm{x}_{1}, \ldots, \mathrm{x}_{n} \exists \mathrm{y}_{1}, \ldots, \mathrm{y}_{m} s_{0}^{1} P_{0}^{1}\left(z_{1,0}^{1}, z_{2,0}^{1}\right) \wedge \ldots \wedge s_{0}^{l(0)} P_{0}^{l(0)}\left(z_{1,0}^{l(0)}, z_{2,0}^{l(0)}\right) \Rightarrow \\
\bigvee_{i=1}^{k} s_{i}^{1} P_{i}^{1}\left(z_{1, i}^{1}, z_{2, i}^{1}\right) \wedge \ldots \wedge s_{0}^{l(i)} P_{0}^{l(i)}\left(z_{1, i}^{l(i)}, z_{2, i}^{l(i)}\right)
\end{gathered}
$$

where

1. each $P_{i}^{j}$ belongs to $\{=, \mathrm{R}\}$; each $s_{i}^{j}$ belongs to $\{\lambda, \neg\}$;

2. each $z_{\alpha, 0}^{\beta}(1 \leq \alpha \leq 2,1 \leq \beta \leq l(0))$ belongs to $\left\{\mathrm{x}_{1}, \ldots, \mathrm{x}_{n}\right\}$;

3. each $z_{\alpha, i}^{\beta}(1 \leq \alpha \leq 2,1 \leq i \leq k, 1 \leq \beta \leq l(i))$ belongs to $\left\{\mathrm{x}_{1}, \ldots, \mathrm{x}_{n}, \mathrm{y}_{1}, \ldots, \mathrm{y}_{m}\right\}$.

We shall now define the $(\psi)$-rule that mimicks the syntactic structure of $\psi$. For any $\mathrm{i}, \mathrm{j} \in \mathrm{For}_{0}^{N}$, for any $s, s^{\prime} \in\{\lambda, \neg\}$ such that $s \neq s^{\prime}$ and for any $P \in\{=, \mathrm{R}\}$, let us define the formula $\Sigma(s P, \mathbf{i}, \mathbf{j})$ as follows:

$$
\Sigma(s P, \mathrm{i}, \mathrm{j}) \stackrel{\text { def }}{=}\left\{\begin{array}{l}
\mathrm{i} \Rightarrow s^{\prime} G \neg \mathrm{j} \text { if } P=\mathrm{R} ; \\
\mathrm{i} \Rightarrow s \mathrm{j} \text { otherwise. }
\end{array}\right.
$$

Roughly speaking, a literal $s P\left(\mathrm{x}_{k}, \mathrm{x}_{k^{\prime}}\right)$ in $\psi$ shall be encoded by $\Sigma\left(s P, \mathbf{i}_{k}, \dot{i}_{k^{\prime}}\right)$. For any formula $\psi$ in $\Phi$, we add the $(\psi)$-rule in Figure 4 to $G \mathcal{L}$. The conditions

$$
\frac{(\Gamma \vdash \Delta) \otimes \sigma_{1} \quad \ldots \quad(\Gamma \vdash \Delta) \otimes \sigma_{k}}{(\Gamma \vdash \Delta) \otimes \sigma_{0}}(\psi)
$$

1. $\sigma_{0}=\Sigma\left(s_{0}^{1} P_{0}^{1}, \mathbf{i}_{1,0}^{1}, \mathbf{i}_{2,0}^{1}\right), \ldots, \Sigma\left(s_{0}^{l(0)} P_{0}^{l(0)}, \mathbf{i}_{1,0}^{l(0)}, i_{2,0}^{l(0)}\right)$;

2. for $1 \leq u \leq k, \sigma_{u}=\sigma_{0} . \Sigma\left(s_{u}^{1} P_{u}^{1}, \mathbf{i}_{1, u}^{1}, \dot{i}_{2, u}^{1}\right) \ldots \Sigma\left(s_{u}^{l(u)} P_{u}^{l(u)}, \mathbf{i}_{1, u}^{l(u)}, \mathbf{i}_{2, u}^{l(u)}\right)$;

3. for any $\alpha, \alpha^{\prime} \in\{1,2\}, q, q^{\prime} \in\{1, \ldots, k\}, r \in\{1, \ldots l(q)\}$ and $r^{\prime} \in\left\{1, \ldots l\left(q^{\prime}\right)\right\}$,

(a) $z_{\alpha, q}^{r}=z_{\alpha^{\prime}, q^{\prime}}^{r^{\prime}}$ iff $\mathbf{i}_{\alpha, q}^{r}=\dot{i}_{\alpha^{\prime}, q^{\prime}}^{r^{\prime}}$;

(b) if $z_{\alpha, q}^{r}$ is equal to some $\mathrm{y}_{i}$, then $\dot{i}_{\alpha, q}^{r}$ does not occur in the conclusion.

Fig. 4. $(\psi)$-rule for $\psi \in \Phi$

1. and 2. in Figure 4 relate the $(\psi)$-rule with the structure of $\psi$ (without taking care of the variables). Condition 3.(a) roughly states that each variable occurring in $\psi$ corresponds to a unique nominal in the application of the $(\psi)$-rule. 
Condition 3.(b) states that the nominals corresponding to the $\mathrm{y}_{i}$ 's are new on the branch. The $(\psi)$-rule can be viewed as a generalization of the " $\rho$-rule" in [Bal98] and of the "Horn relational rule" in [BMV97,BMV98]. More generally, the $(\psi)$-rules merely encodes the logical consequence relation of the first-order relational theory of $\mathcal{L}$ (as also done in [Gen92]). Furthermore, since the definition of the $(\psi)$-rules is purely syntactic, it is not guaranteed that for logics $\mathcal{L}, \mathcal{L}^{\prime}$ in $\mathcal{C}_{\Pi_{2}^{0}}$ characterized by $\Phi$ and $\Phi^{\prime}$ respectively, if $\Phi$ and $\Phi^{\prime}$ define the same class of frames, then $G \mathcal{L}$ and $G \mathcal{L}^{\prime}$ have exactly the same rules.

Example 1. Let $\mathcal{L}_{\neq} \stackrel{\text { def }}{=}\left\langle\operatorname{NTL}(G, H), \mathcal{C}_{\neq}\right\rangle$be the nominal tense logic such that $\Phi \stackrel{\text { def }}{=}\{\forall \mathrm{x}, \mathrm{y} \mathrm{R}(\mathrm{x}, \mathrm{y}) \Rightarrow \neg(\mathrm{x}=\mathrm{y}), \forall \mathrm{x}, \mathrm{y} \neg(\mathrm{x}=\mathrm{y}) \Rightarrow \mathrm{R}(\mathrm{x}, \mathrm{y})\}$. The tense operators $G$ and $H$ are actually equivalent and $G$ is merely the difference modal operator $[\neq]$. The rules of $G \mathcal{L}_{\neq}$are those in Figures $1-3$ plus the rules defined from $\Phi$ :

$$
\frac{\Gamma \vdash \Delta, \mathrm{i} \Rightarrow \mathrm{j}, \mathrm{i} \Rightarrow G \neg \mathrm{j}}{\Gamma \vdash \Delta, \mathrm{i} \Rightarrow G \neg \mathrm{j}} \quad \frac{\Gamma \vdash \Delta, \mathrm{i} \Rightarrow G \neg \mathrm{j}, \mathrm{i} \Rightarrow \mathrm{j}}{\Gamma \vdash \Delta, \mathrm{i} \Rightarrow \mathrm{j}}
$$

A proof $\Pi$ in $G \mathcal{L}$ is a tree whose nodes are labelled by sequents satisfying the following conditions: the topmost sequents of $\Pi$ are initial sequents and every sequent of $\Pi$, except the lowest one is an upper sequent of an inference whose lower sequent is also in $\Pi$. A formula $\phi$ is provable in $G \mathcal{L} \stackrel{\text { def }}{\Leftrightarrow}$ there is a proof $\Pi$ in $G \mathcal{L}$ such that $\vdash \phi$ is the lowest sequent of $\Pi$.

\subsection{Soundness, restrictions and completeness}

Lemma 1. Let $\Gamma \vdash \Delta$ be a sequent provable in $G \mathcal{L}$. Then, for any $\mathcal{L}$-model $\mathcal{M}$, for all $\psi \in \Gamma, \psi$ is true in $\mathcal{M}$ implies that $\phi^{\prime}$ is true in $\mathcal{M}$ for some $\phi^{\prime} \in \Delta$.

The proof is by induction on the length of the derivation. It is more standard to prove soundness by using the notion of satisfiability in a model rather than the notion of truth in a model as done here.

Theorem 1. If $\phi \in \operatorname{NTL}(G, H)$ is provable in $G \mathcal{L}$, then $\phi$ is $\mathcal{L}$-valid.

The system $G \mathcal{L}$ is not minimal since for instance, the $(\vdash N O M=)$-rule, the $\left(N O M_{H} \vdash\right)$-rule and the $\left(N O M_{G} \vdash\right)$-rule are derivable from the rest of $G \mathcal{L}$. These rules are included for the sake of symmetry. The system $G \mathcal{L}$ is considerably improved for the mechanization by imposing the restrictions $\left(R_{\text {init }}\right),\left(R_{\text {start }}\right)$, $(R=),\left(R_{\text {no-renaming }}\right),\left(R_{P B}\right),\left(R_{N O M}\right),\left(R_{\text {witness }}\right),\left(R_{\text {sub }}\right)$ and $\left(R_{\psi}\right)$ for $\psi \in \Phi$ defined below. In the rest of the paper, by $G \mathcal{L}$, we mean the calculus with such restrictions. First, any nominal $\mathrm{j}$ that occurs on a branch of a (possibly partial) proof whose root is labeled by $\phi$ is a $p$-name (standing for "implicit prefix") $\stackrel{\text { def }}{\Leftrightarrow}$ $\mathrm{j}$ has been placed on the branch by application of a rule that introduces new nominals. The notion of p-names is similar to that of Skolem constants.

- $\left(R_{\text {init }}\right)$ for the initial sequent is: any $\phi^{\prime}$ occurring in $\Gamma, i \Rightarrow \psi \vdash \Delta, i \Rightarrow \psi$ is of the form $\mathrm{j} \Rightarrow \psi^{\prime \prime}$ where $\mathrm{j}$ is a p-name, $\psi^{\prime \prime}$ is either a subformula of $\phi$ (syntactically) equal to an atomic proposition in the case when $\mathrm{j} \Rightarrow \psi^{\prime \prime}=$ $i \Rightarrow \psi$ or a p-name, or a nominal occurring in the root sequent $\vdash \phi$ or a formula of the form $G \neg j^{\prime}$ with $j^{\prime}$ a p-name. 
- $\left(R_{\text {start }}\right)$ for the (start)-rule is: $\phi$ is not of the form $j \Rightarrow \phi^{\prime}$ where $j$ is a p-name.

- $(R=)$ concerns the rules $($ refl $),($ sym $),($ trans $),(\vdash$ sub $)$ and $(s u b \vdash)$ : all the names $i, j, k$ are p-names.

- $\left(R_{n o-r e n a m i n g}\right)$ is: in $(\vdash G)$ and in $(\vdash H) \psi$ is not a negated p-name.

- $\left(R_{P B}\right)$ is: $i$ is a p-name and $\psi$ is either a subformula in $\phi$ of secondary disjunctive force or $G \neg j$ with $j$ a p-name or a p-name $j$.

- $\left(R_{N O M}\right)$ concerns the rules $(\vdash N O M=),(N O M=\vdash),\left(\vdash N O M_{G}\right),\left(N O M_{G} \vdash\right.$ )$,\left(\vdash N O M_{H}\right),\left(N O M_{H} \vdash\right): i$ and $i^{\prime}$ are p-names whereas $\mathrm{j}$ is not a p-name. - $\left(R_{\text {witness }}\right)$ concerns the rules $(G \vdash)$ and $(H \vdash): i$ and $j$ are p-names.

- $\left(R_{s u b^{\prime}}\right)$ is: in the $\left(s u b^{\prime} \vdash\right)$-rule and the $\left(\vdash s u b^{\prime}\right)$-rule, $i, j$ and $j^{\prime}$ are p-names.

- The restriction $\left(R_{\psi}\right)$ for the $(\psi)$-rule for $\psi \in \Phi$ is: all the nominals occurring in $\sigma_{0}$ are p-names.

The sequent calculus $G \mathcal{L}$ (in its restricted form) has the following separation property: any p-name $i$ occurring in a branch does not occur in a formula $j \Rightarrow \psi$ occurring on the same branch, except when either $j=i$ or $\psi=G \neg i$ or $\psi=i$. This separation property illustrates the control on the use of nominals imposed by the above restrictions.

Theorem 2. If $\phi \in \operatorname{NTL}(G, H)$ is $\mathcal{L}$-valid, then $\phi$ is provable in $G \mathcal{L}$.

The proof of Theorem 2 (using Schütte's method) is based on a similar proof for classical logic. In Section 4, we formally state in which sense $G \mathcal{L}$ is equivalent to a calculus for a fragment of classical logic.

\section{Sequent calculi for fragments of classical logic with relational theories}

In this section, we define a first-order Gentzen-style calculus $G F O L_{\mathcal{L}}$ (based on the calculus KEQ [d'A90, Section 3.5]) such that $G \mathcal{L}$ and $G F O L_{\mathcal{L}}$ can be viewed as syntactic variants. This is the opportunity to formally present (once and for all) how a tableaux calculus can be viewed as a translation into classical logic. Let us briefly recall the translation ST ("Standard Translation") defined in [Ben83,GG93] of nominal tense formulae into the first-order language (here $\mathrm{t}$ is either a variable or a constant): $S T\left(\mathrm{p}_{j}, \mathrm{t}\right) \stackrel{\text { def }}{=} \mathrm{P}_{j}(\mathrm{t}) ; S T\left(\dot{\mathrm{i}}_{j}, \mathrm{t}\right) \stackrel{\text { def }}{=}$ $\mathrm{t}=\mathrm{a}_{j} ; S T(\neg \psi, \mathrm{t}) \stackrel{\text { def }}{=} \neg S T(\psi, \mathrm{t}) ; S T\left(\psi \oplus \phi^{\prime}, \mathrm{t}\right) \stackrel{\text { def }}{=} S T(\psi, \mathrm{t}) \oplus S T\left(\phi^{\prime}, \mathrm{t}\right)$ for $\oplus \in$ $\{\wedge, \Rightarrow\} ; S T(G \psi, \mathrm{t}) \stackrel{\text { def }}{=} \forall \mathrm{x}^{\prime}\left(\mathrm{R}\left(\mathrm{t}, \mathrm{x}^{\prime}\right) \Rightarrow S T\left(\psi, \mathrm{x}^{\prime}\right)\right)$ where $\mathrm{x}^{\prime}$ is a new variable; $S T(H \psi, \mathrm{t}) \stackrel{\text { def }}{=} \forall \mathrm{x}^{\prime}\left(\mathrm{R}\left(\mathrm{x}^{\prime}, \mathrm{t}\right) \Rightarrow S T\left(\psi, \mathrm{x}^{\prime}\right)\right)$ where $\mathrm{x}^{\prime}$ is a new variable. It is known that $\phi$ is $\mathcal{L}$-valid iff $\Phi \Rightarrow \forall \mathrm{x}_{0} S T\left(\phi, \mathrm{x}_{0}\right)$ is FOL-valid. The previous statement assumes that $\Phi$ is a finite conjunction. By contrast, the developments in this section does not assume that $\Phi$ is finite. The rules of the calculus $G F O L_{\mathcal{L}}$ are those presented in Figure 5 - Figure 6 (other rules are added later on). Like the notion of p-name in $G \mathcal{L}$, an individual constant a occurring on a branch is said to be a $p$-constant (or Skolem constant) $\stackrel{\text { def }}{\Leftrightarrow}$ a does not occur in the root sequent of the proof (possibly in construction) and it has been introduced on the branch by a rule putting new constants on the branches. We write $\psi\left(\mathrm{a}_{k}\right)[\operatorname{resp} . \psi(\mathrm{x})]$ to denote the formula whose $\mathrm{a}_{k}$ is a p-constant occurring in it [resp. whose $\mathrm{x}$ is a free individual variable occurring in it.]. 
$\Gamma, \psi \vdash \Delta, \psi$ (initial sequents)

under the proviso: any formula $\phi^{\prime}$ in $\Gamma, \psi, \Delta$ is (1) either a subformula $\psi^{\prime \prime}$ (a) of $\forall \mathrm{x}_{0} S T\left(\phi, \mathrm{x}_{0}\right)$ where a is the unique p-constant in $\psi^{\prime \prime}(\mathrm{a})$ and in the case when $\psi=\psi^{\prime \prime}$, $\psi$ is atomic (2) or a formula $\mathrm{a}=\mathrm{b}$ where $\mathrm{a}$ is a p-constant and $\mathrm{b}$ is either a p-constant or a constant occurring in $\forall \mathrm{x}_{0} S T\left(\phi, \mathrm{x}_{0}\right)$ (3) or a formula $\mathrm{R}(\mathrm{a}, \mathrm{b})$ where a and $\mathrm{b}$ are p-names.

$$
\frac{\Gamma \vdash \Delta, \psi \quad \Gamma, \psi \vdash \Delta}{\Gamma \vdash \Delta}(P B)
$$

where either $\psi$ is a formula of secondary disjunctive force occurring below in the proof containing a unique p-constant or $\psi$ is of the form $\mathrm{a}_{k}=\mathrm{a}_{k^{\prime}}$ or $\mathrm{R}\left(\mathrm{a}_{k}, \mathrm{a}_{k^{\prime}}\right)$ where $\mathrm{a}_{k}$ and $\mathrm{a}_{k^{\prime}}$ are p-constants.

$$
\frac{\vdash S T\left(\phi, \mathrm{a}_{k}\right)}{\vdash \forall \mathrm{x}_{0} S T\left(\phi, \mathrm{x}_{0}\right)}(\text { start })
$$

the application of (start) is under the proviso that $\mathrm{a}_{k}$ does not occur in $S T(\phi, \mathrm{x})$ (or equivalently, $\dot{i}_{k}$ does not occur in $\left.\phi\right)$ and $\forall \mathrm{x}_{0} S T\left(\phi, \mathrm{x}_{0}\right)$ does not contain p-constants.

Fig. 5. First bunch of rules for $G F O L_{\mathcal{L}}$

For instance, the rules $(\forall \vdash)_{1}$ and $(\forall \vdash)_{2}$ can be seen as derived rules in the calculus KEQ [d'A90] using the rules from KEQ recalled below

$$
\frac{\Gamma, \forall \mathrm{x} \phi(\mathrm{x}), \phi(\mathrm{a}) \vdash \Delta}{\Gamma, \forall \mathrm{x} \phi(\mathrm{x}) \vdash \Delta} \quad \frac{\Gamma, \phi_{1}, \phi_{2}, \vdash \Delta}{\Gamma, \phi_{1}, \phi_{1} \Rightarrow \phi_{2}, \vdash \Delta}
$$

This explains why the universal quantification in modal logic can be naturally encoded in KEQ .

Let $\sigma$ be a finite sequence of formulas of the form $\mathrm{R}\left(\mathrm{a}, \mathrm{a}^{\prime}\right), \neg \mathrm{R}\left(\mathrm{a}, \mathrm{a}^{\prime}\right), \mathrm{a}=\mathrm{a}^{\prime}$, $\neg\left(\mathrm{a}=\mathrm{a}^{\prime}\right)$. We define the sequent $(\Gamma \vdash \Delta) \otimes^{\prime} \sigma$ inductively as follows:

$$
\begin{aligned}
& -(\Gamma \vdash \Delta) \otimes^{\prime} \lambda \stackrel{\text { def }}{=} \Gamma \vdash \Delta ;(\Gamma \vdash \Delta) \otimes^{\prime} \mathrm{a}=\mathrm{a}^{\prime} \cdot \sigma^{\prime} \stackrel{\text { def }}{=}\left(\Gamma, \mathrm{a}=\mathrm{a}^{\prime} \vdash \Delta\right) \otimes^{\prime} \sigma^{\prime} ; \\
& -(\Gamma \vdash \Delta) \otimes^{\prime} \neg \mathrm{R}\left(\mathrm{a}, \mathrm{a}^{\prime}\right) \cdot \sigma^{\prime} \stackrel{\text { def }}{=}\left(\Gamma \vdash \Delta, \mathrm{R}\left(\mathrm{a}, \mathrm{a}^{\prime}\right)\right) \otimes^{\prime} \sigma^{\prime} ; \\
& -(\Gamma \vdash \Delta) \otimes^{\prime} \neg\left(\mathrm{a}=\mathrm{a}^{\prime}\right) \cdot \sigma^{\prime} \stackrel{\text { def }}{=}\left(\Gamma \vdash \Delta, \mathrm{a}=\mathrm{a}^{\prime}\right) \otimes^{\prime} \sigma^{\prime} ; \\
& -(\Gamma \vdash \Delta) \otimes^{\prime} \mathrm{R}\left(\mathrm{a}, \mathrm{a}^{\prime}\right) \cdot \sigma^{\prime} \stackrel{\text { def }}{=}\left(\Gamma, \mathrm{R}\left(\mathrm{a}, \mathrm{a}^{\prime}\right) \vdash \Delta\right) \otimes^{\prime} \sigma^{\prime} .
\end{aligned}
$$

Let $\psi$ be a restricted $\Pi_{2}^{0}$-formula in $\Phi$ (we use the notations from Section 3 ). The rule associated to $\psi$ is presented in Figure 7.

By construction, the calculi $G \mathcal{L}$ and $G F O L_{\mathcal{L}}$ have (almost) the same amount of rules and there is a natural correspondence between the rules of $G \mathcal{L}$ and $G F O L_{\mathcal{L}}$. For instance, the $(\forall \vdash)_{1}$-rule in $G F O L_{\mathcal{L}}$ correspond to the $(G \vdash)$-rule in $G \mathcal{L}$ and the $(s u b \vdash)$-rule and $\left(s u b^{\prime} \vdash\right)$-rule in $G \mathcal{L}$ correspond to the $\left(s u b_{f o l}\right)$ rule in $G F O L_{\mathcal{L}}$.

Let $\phi \in$ For and $\Pi$ be a proof of $\forall \mathrm{x}_{0} S T\left(\phi, \mathrm{x}_{0}\right)$ in $G F O L_{\mathcal{L}}$. By induction on the length of $\Pi$ one can show that any formula $\psi$ occurring in $\Pi$ has at most two p-constants occurring in it. Moreover, if $\psi$ is not an atomic formula 


$$
\begin{gathered}
\frac{\Gamma, \phi \vdash \Delta}{\Gamma \vdash \Delta, \neg \phi}(\vdash \neg) \quad \frac{\Gamma \vdash \Delta, \phi}{\Gamma, \neg \phi \vdash \Delta}(\neg \vdash) \quad \frac{\Gamma, \phi_{1} \vdash \Delta, \phi_{2}}{\Gamma \vdash \Delta, \phi_{1} \Rightarrow \phi_{2}}(\vdash \Rightarrow) \\
\frac{\Gamma, \phi_{1}, \phi_{2} \vdash \Delta}{\Gamma, \phi_{1}, \phi_{1} \Rightarrow \phi_{2} \vdash \Delta}(\Rightarrow \vdash)_{1} \quad \frac{\Gamma \vdash \Delta, \phi_{1}, \phi_{2}}{\Gamma, \phi_{1} \Rightarrow \phi_{2} \vdash \Delta, \phi_{2}}(\Rightarrow \vdash)_{2} \\
\frac{\Gamma, \phi \vdash \Delta, \psi}{\Gamma, \phi \vdash \Delta, \phi \wedge \psi}(\vdash \wedge)_{1} \quad \frac{\Gamma, \psi \vdash \Delta, \phi}{\Gamma, \psi \vdash \Delta, \phi \wedge \psi}(\vdash \wedge)_{2} \quad \frac{\Gamma, \phi, \psi \vdash \Delta}{\Gamma, \phi \wedge \psi \vdash \Delta}(\wedge \vdash) \\
\frac{\Gamma, \mathrm{R}(\mathrm{a}, \mathrm{b}), \forall \mathrm{x}(\mathrm{R}(\mathrm{a}, \mathrm{x}) \Rightarrow \phi(\mathrm{x})), \phi(\mathrm{b}) \vdash \Delta}{\Gamma, \mathrm{R}(\mathrm{a}, \mathrm{b}), \forall \mathrm{x}(\mathrm{R}(\mathrm{a}, \mathrm{x}) \Rightarrow \phi(\mathrm{x})) \vdash \Delta}(\forall \vdash)_{1} \frac{\Gamma, \mathrm{R}(\mathrm{b}, \mathrm{a}), \forall \mathrm{x}(\mathrm{R}(\mathrm{x}, \mathrm{a}) \Rightarrow \phi(\mathrm{x})), \phi(\mathrm{b}) \vdash \Delta}{\Gamma, \mathrm{R}(\mathrm{b}, \mathrm{a}), \forall \mathrm{x}(\mathrm{R}(\mathrm{x}, \mathrm{a}) \Rightarrow \phi(\mathrm{x})) \vdash \Delta}(\forall \vdash)_{2} \\
\frac{\Gamma, \mathrm{R}(\mathrm{a}, \mathrm{b}), \vdash \Delta, \phi(\mathrm{b})}{\Gamma \vdash \Delta, \forall \mathrm{x}(\mathrm{R}(\mathrm{a}, \mathrm{x}) \Rightarrow \phi(\mathrm{x}))}(\vdash \forall)_{1} \quad \frac{\Gamma, \mathrm{R}(\mathrm{b}, \mathrm{a}), \vdash \Delta, \phi(\mathrm{b})}{\Gamma \vdash \Delta, \forall \mathrm{x}(\mathrm{R}(\mathrm{x}, \mathrm{a}) \Rightarrow \phi(\mathrm{x}))}(\vdash \forall)_{2}
\end{gathered}
$$

under the proviso that $\mathrm{b}$ does not occur in the lower sequent.

$$
\begin{gathered}
\frac{\Gamma, \mathrm{a}_{k_{1}}=\mathrm{a}_{k}, \mathrm{a}_{k_{2}}=\mathrm{a}_{k}, \mathrm{a}_{k_{1}}=\mathrm{a}_{k_{2}} \vdash \Delta}{\Gamma, \mathrm{a}_{k_{1}}=\mathrm{a}_{k}, \mathrm{a}_{k_{2}}=\mathrm{a}_{k} \vdash \Delta}\left(N O M_{=}^{\prime} \vdash\right) \\
\frac{\Gamma, \psi\left(\mathrm{a}_{k_{1}}\right) \vdash \Delta, \psi\left(\mathrm{a}_{k_{2}}\right), \mathrm{a}_{k_{1}}=\mathrm{a}_{k_{2}}}{\Gamma, \psi\left(\mathrm{a}_{k_{1}}\right) \vdash \Delta, \psi\left(\mathrm{a}_{k_{2}}\right)}\left(\vdash O M_{=}^{\prime}\right) \\
\frac{\Gamma, \forall \mathrm{x}\left(\mathrm{R}\left(\mathrm{a}_{k_{1}}, \mathrm{x}\right) \Rightarrow \psi(\mathrm{x})\right) \vdash \Delta, \psi\left(\mathrm{a}_{k_{2}}\right), \mathrm{R}\left(\mathrm{a}_{k_{1}}, \mathrm{a}_{k_{2}}\right)}{\Gamma, \forall \mathrm{x}\left(\mathrm{R}\left(\mathrm{a}_{k_{1}}, \mathrm{x}\right) \Rightarrow \psi(\mathrm{x})\right) \vdash \Delta, \psi\left(\mathrm{a}_{k_{2}}\right)}\left(N O M_{G}^{\prime} \vdash\right) \\
\frac{\Gamma, \mathrm{a}_{k_{1}}=\mathrm{a}_{k}, \mathrm{R}\left(\mathrm{a}_{k_{2}}, \mathrm{a}_{k_{1}}\right) \vdash \Delta, \forall \mathrm{x}\left(\mathrm{R}\left(\mathrm{a}_{k_{2}}, \mathrm{x}\right) \Rightarrow \neg\left(\mathrm{x}=\mathrm{a}_{k}\right)\right)}{\Gamma, \mathrm{a}_{k_{1}}=\mathrm{a}_{k} \vdash \Delta, \forall \mathrm{x}\left(\mathrm{R}\left(\mathrm{a}_{k_{2}}, \mathrm{x}\right) \Rightarrow \neg\left(\mathrm{x}=\mathrm{a}_{k}\right)\right)}\left(\vdash O M_{G}^{\prime}\right) \\
\frac{\Gamma, \forall \mathrm{x}\left(\mathrm{R}\left(\mathrm{a}_{k_{1}}, \mathrm{x}\right) \Rightarrow \psi(\mathrm{x})\right) \vdash \Delta, \psi\left(\mathrm{a}_{k_{2}}\right), \mathrm{R}\left(\mathrm{a}_{k_{2}}, \mathrm{a}_{k_{1}}\right)}{\Gamma, \forall \mathrm{x}\left(\mathrm{R}\left(\mathrm{x}, \mathrm{a}_{k_{1}}\right) \Rightarrow \psi(\mathrm{x})\right) \vdash \Delta, \psi\left(\mathrm{a}_{k_{2}}\right)}\left(N O M_{H}^{\prime} \vdash\right) \\
\frac{\Gamma, \mathrm{a}_{k_{1}}=\mathrm{a}_{k}, \mathrm{R}\left(\mathrm{a}_{k_{1}}, \mathrm{a}_{k_{2}}\right) \vdash \Delta, \forall \mathrm{x}\left(\mathrm{R}\left(\mathrm{a}_{k_{2}}, \mathrm{x}\right) \Rightarrow \neg\left(\mathrm{x}=\mathrm{a}_{k}\right)\right)}{\Gamma, \mathrm{a}_{k_{1}}=\mathrm{a}_{k} \vdash \Delta, \forall \mathrm{x}\left(\mathrm{R}\left(\mathrm{x}, \mathrm{a}_{k_{2}}\right) \Rightarrow \neg\left(\mathrm{x}=\mathrm{a}_{k}\right)\right)}\left(\vdash N M_{H}^{\prime}\right)
\end{gathered}
$$

in the above rules, $\mathbf{a}_{k_{1}}$ and $\mathbf{a}_{k_{2}}$ are p-constants and $\mathbf{a}_{k}$ is not a p-constant.

$$
\begin{gathered}
\frac{\Gamma, \mathrm{a}=\mathrm{a} \vdash \Delta}{\Gamma \vdash \Delta} \text { a occurs in } \Gamma, \Delta \quad \frac{\Gamma, \mathrm{a}=\mathrm{b}, \mathrm{b}=\mathrm{a}, \vdash \Delta}{\Gamma, \mathrm{a}=\mathrm{b} \vdash \Delta} \frac{\Gamma, \mathrm{a}=\mathrm{b}, \mathrm{b}=\mathrm{b}^{\prime}, \mathrm{a}=\mathrm{b}^{\prime}, \vdash \Delta}{\Gamma, \mathrm{a}=\mathrm{b}, \mathrm{b}^{\prime} \mathrm{b}^{\prime} \vdash \Delta} \\
\frac{\Gamma, \mathrm{a}=\mathrm{b} \vdash \Delta, \phi(\mathrm{a}), \psi(\mathrm{b})}{\Gamma, \mathrm{a}=\mathrm{b} \vdash \Delta, \psi(\mathrm{a})}\left(\vdash s u b_{f o l}\right) \quad \frac{\Gamma, \mathrm{a}=\mathrm{b}, \psi(\mathrm{a}), \psi(\mathrm{b}) \vdash \Delta}{\Gamma, \mathrm{a}=\mathrm{b}, \psi(\mathrm{a}) \vdash \Delta}\left(s u b_{f o l} \vdash\right)
\end{gathered}
$$

In the above rules, $\mathrm{a}, \mathrm{b}$ and $\mathrm{b}^{\prime}$ are $\mathrm{p}$-constants

Fig. 6. Common core of (introduction rules) for $G F O L_{\mathcal{L}}$ 


$$
\frac{(\Gamma \vdash \Delta) \otimes^{\prime} \sigma_{1} \quad \ldots \quad(\Gamma \vdash \Delta) \otimes^{\prime} \sigma_{k}}{(\Gamma \vdash \Delta) \otimes^{\prime} \sigma_{0}}(\psi)
$$

1. $\sigma_{0}=s_{0}^{1} P_{0}^{1}\left(\mathrm{a}_{1,0}^{1}, \mathrm{a}_{2,0}^{1}\right), \ldots, s_{0}^{l(0)} P_{0}^{l(0)}\left(\mathrm{a}_{1,0}^{l(0)}, \mathrm{a}_{2,0}^{l(0)}\right)$ and all the constants in $\sigma_{0}$ are $\mathrm{p}$ constants;

2. for $1 \leq u \leq k, \sigma_{u}=\sigma_{0} \cdot s_{u}^{1} P_{u}^{1}\left(\mathrm{a}_{1, u}^{1}, \mathrm{a}_{2, u}^{1}\right), \ldots, s_{0}^{l(u)} P_{0}^{l(u)}\left(\mathrm{a}_{1, u}^{l(u)}, \mathrm{a}_{2, u}^{l(u)}\right)$;

3. for any $\alpha, \alpha^{\prime} \in\{1,2\}, q, q^{\prime} \in\{1, \ldots, k\}, r \in\{1, \ldots l(q)\}$ and $r^{\prime} \in\left\{1, \ldots l\left(q^{\prime}\right)\right\}$,

(a) $z_{\alpha, q}^{r}=z_{\alpha^{\prime}, q^{\prime}}^{r^{\prime}}$ iff $\mathrm{a}_{\alpha, q}^{r}=\mathrm{a}_{\alpha^{\prime}, q^{\prime}}^{r^{\prime}}$;

(b) if $z_{\alpha, q}^{r}$ is equal to some $\mathrm{y}_{i}$, then $\mathrm{a}_{\alpha, q}^{r}$ does not occur in the conclusion.

Fig. 7. $(\psi)$-rule for $\psi \in \Phi$

whose predicate symbol is binary, then exactly one p-constant occurs in $\psi$ unless $\psi$ is the root formula $\forall \mathrm{x}_{0} S T\left(\phi, \mathrm{x}_{0}\right)$ itself. This is reminiscent of the facts that in standard modal logic, one can deal with only one world at a time and two individual variables are sufficient for encoding the quantification $\square$ in first-order logic. Theorem 3 below helps understanding the relationships between $G \mathcal{L}$ and $G F O L_{\mathcal{L}}$.

Theorem 3. (I) Let $\Pi$ be a proof of $\phi$ in $G \mathcal{L}$. Then, there is a proof $\Pi^{\prime}$ of $\forall \mathrm{x}_{0} S T\left(\phi, \mathrm{x}_{0}\right)$ in $G F O L_{\mathcal{L}}$ such that $\left|\Pi^{\prime}\right|$ is in $\mathcal{O}(|\Pi|)$.

(II) Let $\Pi$ be a proof of $\forall \mathrm{x}_{0} S T\left(\phi, \mathrm{x}_{0}\right)$ in $G F O L_{\mathcal{L}}$ for some nominal tense formula $\phi$. Then, there is a proof $\Pi^{\prime}$ of $\phi$ in $G \mathcal{L}$ such that $\left|\Pi^{\prime}\right|$ is in $\mathcal{O}(|\Pi|)$.

\section{Concluding remarks}

The results of the previous sections can be extended to the polymodal case. Indeed, it is easy to consider for some countable set $I$ of "modal terms", the family $\left\{G_{i}: i \in I\right\} \cup\left\{H_{i}: i \in I\right\}$ of tense operators by appropriately considering polymodal Kripke models. The class $\mathcal{C}_{\Pi_{0}^{2}}^{I}$ is defined as the class of polymodal logics such that the class of frames is determined by a (possibly infinite) set of restricted $\Pi_{2}^{0}$-formulae over the vocabulary containing $\left\{\mathrm{R}_{i}: i \in I\right\}$. This extension does not generate any new technical problems and it is quite powerful as shown below. Let $I_{0}=\left\{\mathrm{c}_{0}, \ldots, \mathrm{c}_{i}, \ldots\right\}$ be a set of modal constants and $I$ be the set of modal terms $t$ inductively defined as follows: $t::=i d\left|c_{i}\right|-t \mid$ $t^{-1}\left|t_{1} \cup t_{2}\right| t_{1} \cap t_{2} \mid t_{1} \circ t_{2}$ for $c_{i} \in I_{0}$. We wish to interpret the operators $-,^{-1}, \cup, \cap$ and $\circ$ and the identity constant id as in the Relation Calculus. Although it is known that the Relation Calculus can be translated in classical logic, surprisingly, we can also capture such a semantics in our framework using only restricted $\Pi_{2}^{0}$-formulae. So, by using our framework we can deal with nominal (poly)tense logics admitting the operators $-,{ }^{-1}, \cap, \cup, \circ$ and this is done uniformly ${ }^{6}$ (this list of operators is not exhaustive). By contrast, in [Bal98, Chapter VI], only the operators $\cup$ and $\circ$ and the constant id are treated.

In this paper, we defined sequent calculi for nominal tense logics. The idea of using "implicit prefixes" in the calculi, due to [Kon97], allows a great flexibility

\footnotetext{
${ }^{6}$ Numerous description logics can be also treated within our framework
} 
and we have been able to consider most of the classes of modal frames first-order definable that can be found in the literature. Using standard correspondences, it is easy to define tableaux calculi for nominal tense logics from our sequent-style calculi. Extensions of the calculi to cope with the logical consequence relations are also possible. Moreover, by appropriately modifying the (start)-rule, one can deal with finite configuration in the sense of [Rus96, Chapters 2 and 3]. Similarly, prefixed calculi (either sequent-based or tableaux-based) could be easily defined for the corresponding (non nominal) tense logics. Because of lack of space, such developments are omitted here but they are not difficult to derive from the present paper. Similarly, the design of decision procedures from our calculi was out of the scope of this paper but it is a question worth being investigated in the future.

The adequateness of our framework for mechanization cannot be stated without further investigations although it seems theoretically appealing (see for instance in Section 3.3 how the application of rules can be restricted). There is no reason to be overly optimistic since we have shown that the non prefixed sequent calculi are syntactic variants of restricted calculi for classical logic (augmented with relational theories). This property is shared by numerous calculi from the literature. As a conclusion, it is an open question whether any general framework defining sequent-style proof systems for modal (or nominal tense, ...) logics characterized by first-order definable classes of modal frames (take for instance $\left.\mathcal{C}_{\Pi_{2}^{0}}\right)$ is bound to define syntactic variants of calculi for fragments of classical logic augmented with relational theories.

Acknowledgments The author thanks Rajeev Goré for suggestions about a preliminary version of this work and for many discussions about prefixed tableaux and related matters while being an International ARC Fellow at the A.R.P. Thanks are also due to the referees for many useful suggestions and remarks and to Nicolas Peltier for reading a preliminary version.

\section{References}

[Bal98] M. Baldoni. Normal Multimodal Logics: Automated Deduction and Logic Programming. PhD thesis, Università degli Studi di Torino, 1998.

[Ben83] J. van Benthem. Modal logic and classical logic. Bibliopolis, 1983.

[BG97] B. Beckert and R. Goré. Free variable tableaux for propositional modal logics. In TABLEAUX'97, pages 91-106. LNAI 1227, Springer, 1997.

[BG98] N. Bonnette and R. Goré. A labelled sequent systems for tense logic $\mathrm{K}_{t}$. In Australian Joint Conference of Articifial Intelligence, pages 71-82. LNAI 1502, Springer, 1998.

[Bla93] P. Blackburn. Nominal tense logic. Notre Dame Journal of Formal Logic, 34(1):56-83, 1993.

[Bla98] P. Blackburn. Internalizing labeled deduction. Technical Report 102, Computerlinguistik, Universität des Saarlandes, 1998.

[BMV97] D. Basin, S. Matthews, and L. Viganò. Labelled propositional modal logics: Theory and practice. J. of Logic and Computation, 7(6):685-717, 1997.

[BMV98] D. Basin, S. Matthews, and L. Viganò. Natural deduction for nonclassical logics. Studia Logica, 60(1):119-160, 1998. 
[Boo84] G. Boolos. Don't eliminate cut. J. of Philosophical Logic, 13:373-378, 1984.

[CFdCGH97] M. Castilho, L. Fariñas del Cerro, O. Gasquet, and A. Herzig. Modal tableaux with propagation rules and structural rules. Fundamenta Informaticae, 32(3/4):281-297 1997.

[d'A90] M. d'Agostino. Investigations into the complexity of some propositional calculi. PhD thesis, Oxford University Computing Laboratory, 1990.

[DG99] S. Demri and R. Goré. Cut-free display calculi for nominal tense logics. In this volume, 1999.

[dM94] M. d'Agostino and M. Mondadori. The taming of the cut. Classical refutations with analytic cut. J. of Logic and Computation, 4(3):285319, 1994

[Fit83] M. Fitting. Proof methods for modal and intuitionistic logics. D. Reidel Publishing Co., 1983.

[Gab96] D. Gabbay. Labelled Deductive Systems. Oxford University Press, 1996.

[Gen92] I. Gent. Analytic proof systems for classical and modal logics of restricted quantification. PhD thesis, University of Warwick, 1992.

[GG93] G. Gargov and V. Goranko. Modal logic with names. J. of Philosophical Logic, 22(6):607-636, 1993.

[GHM98] H. Ganzinger, U. Hustadt, and R. Meyer and C. Schmidt. A resolutionbased decision procedure for extensions of K4. In 2nd Workshop on Advances in Modal Logic, 1998. to appear.

[Gor99] R Goré. Tableaux methods for modal and temporal logics. In Handbook of Tableaux Methods. Kluwer, Dordrecht, 1999. to appear.

[Gov95] G. Governatori. Labelled tableaux for multi-modal logics. In TABLEAUX-4, pages 79-94. LNAI 918, Springer, 1995.

[Heu98] A. Heuerding. Sequent Calculi for Proof Search in Some Modal Logics. PhD thesis, University of Bern, 1998.

[Hud96] J. Hudelmaier. Improved decision procedures for the modal logics K, KT and S4. In CSL'95, pages 320-334. LNCS 1092, Springer, 1996.

[Kon97] B. Konikowska. A logic for reasoning about relative similarity. Studia Logica, 58(1):185-226, 1997.

[Kra96] M. Kracht. Power and weakness of the modal display calculus. In H. Wansing, editor, Proof theory of modal logic, pages 93-121. Kluwer Academic Publishers, 1996.

[Kri63] S. Kripke. Semantical analysis of modal logic I: normal modal propositional calculi. Zeitschrift für Mathematik Logik und Grundlagen der Mathematik, 9:67-96, 1963.

[Mas94] F. Massacci. Strongly analytic tableaux for normal modal logics. In $C A D E-12$, pages 723-737. Springer, LNAI 814, 1994.

[Ogn94] Z. Ognjanović. A tableau-like proof procedure for normal modal logics. TCS, 129:167-186, 1994.

[Pri57] A. Prior. Time and Modality. Clarendon Press, Oxford, 1957.

[RU71] N. Rescher and A. Urquhart. Temporal Logic. Springer-Verlag, 1971.

[Rus96] A. Russo. Modal logics as labelled deductive systems. PhD thesis, Imperial College, London, 1996.

[Tza99] M. Tzakova. Tableau calculi for hybrid logics. In this volume, 1999.

[Wa190] L. Wallen. Automated Deduction in Nonclassical Logics. MIT Press, 1990. 